disturbance, 9) liver dysfunction (pemoline), 10) treatment with other medications eg. clonidine, MAO inhibitors.

\title{
OPPOSITIONAL DEFIANT DISORDER, CONDUCT, AND ADHD
}

The link between oppositional defiant disorder (ODD) and conduct disorder (CD) was evaluated in 140 children with attention-deficit hyperactivity disorder (ADHD) and 120 normal controls examined at baseline and 4 years later, in midadolescence, at the Pediatric Psychopharmacology Unit, Psychiatric Service, Massachusetts General Hospital, Boston, MA. Of ADHD children, $65 \%$ had comorbid ODD and $22 \%$ had CD at baseline. Of ODD children, $32 \%$ had comorbid CD. Children with CD also had ODD that preceded CD by several years. Children with both ODD and CD had more severe symptoms of ODD, more psychiatric disorders, more bipolar disorder, and more abnormal behavior scores compared to ADHD children without comorbidity. Risk of CD at 4-year follow-up was not increased in children with ODD without CD at baseline. Two subtypes of ODD associated with ADHD were evident: one prodromal to $\mathrm{CD}$ and one that is not. (Biederman $\mathrm{J}$ et al. Is childhood oppositional defiant disorder a precursor to adolescent conduct disorder? Findings from a four-year follow-up study of children with ADHD. I Am Acad Child Adolesc Psychiatry Sept 1996;35:1193-1204). (Reprints: Dr Biederman, Pediatric Psychopharmacology Unit (ACC 725), Massachusetts General Hospital, Fruit Street, Boston, MA 02114).

COMMENT. The majority of ODD children with ADHD do not have comorbid CD, whereas CD is almost always comorbid with ODD which precedes the onset of $C D$ by several years. The majority of children with ADHD and CD had developed $C D$ before age 12 years. Adolescent onset $C D$ is rare. CD with $\mathrm{ADHD}$ is associated with higher frequency of substance abuse in adolescence, and higher levels of anxiety disorders and mood disorders. Two ODD subtypes, one prodromal to $\mathrm{CD}$ and one without, have different outcomes.

\section{LEARNING AND BEHAVIOR DISORDERS}

\section{NEURAL BASIS OF DYSLEXIA}

Whole-head magnetoencephalography (MEG) was employed to track noninvasively the cortical activation sequences during visual word recognition in 6 adult dyslexic and 8 control subjects examined at the Brain Research Unit, Helsinki University of Technology, Espoo; and the Departments of Psychology and Radiology, University of Helsinki, Helsinki, Finland. Significant differences between the two groups were found for the time window 0 to $200 \mathrm{msec}$ after single word presentation in the left inferior temporo-occipital cortex, for 200 to $400 \mathrm{msec}$ in the left temporal lobe, and for 0 to $400 \mathrm{msec}$ in the left inferior frontal lobe. Considerable interindividual variability was shown for spatiotemporal activation patterns. Dyslexics failed to activate the left inferior temporo-occipital cortex within $200 \mathrm{msec}$ after word presentation. The left temporal lobe, including Wernicke's area, a region associated with phonological aspects of language, was strongly involved in controls but not in dyslexics. Dyslexics activated, instead, the left inferior frontal lobe, involving Broca's area, whereas activation of the right motor/premotor cortex, present in controls, was absent in dyslexics. Perception of words as specific units was impaired in dyslexics. (Salmelin R et al. Impaired visual word processing in dyslexia revealed with 